\title{
ON STABLE VECTOR BUNDLES OVER REAL PROJECTIVE SPACES
}

\author{
DAVID HANDEL ${ }^{1}$
}

If $X$ is a connected, finite $C W$-complex, we can define $(K O) \sim(X)$ to be $[X, B O]$ (base-point preserving homotopy classes of maps). Recall [2] that if $x \in(K O)^{\sim}(X)$, the geometrical dimension of $x$ (abbreviated g.dim $x$ ) can be defined to be the smallest nonnegative integer $k$ such that a representative of $x$ factors through $B O(k)$. If $\xi$ is a vector bundle over $X$, the class in $(K O)^{\sim}(X)$ of a classifying map for $\xi$ is called the stable class of $\xi$.

Let $P^{n}$ denote real projective $n$-space, and let $x_{n} \in(K O)^{\sim}\left(P^{n}\right)$ denote the stable class of the canonical line bundle $\gamma_{n}$ over $P^{n}$.

If $m$ is a positive integer, let $\phi(m)$ denote the number of integers $k$ such that $0<k \leqq m$ and $k \equiv 0,1,2,4(\bmod 8)$. The purpose of this note is to prove the following theorem:

Theorem. Let $n-2<2 m<2 n$. Then for any integers $r, s$, g.dim $\left(2^{\phi(m)} r s x_{n}\right) \leqq \max \left\{m+1, g \cdot \operatorname{dim}\left(2^{\phi(m)} r x_{n}\right)\right\}$.

Applications are given below. We first need two lemmas.

Lemma 1. Let $X \supset Y$ be a pair of finite $C W$-complexes, and let $p: X \rightarrow X / Y$ be the natural projection. Let $x \in(K O) \sim(X)$ be in the image of $p^{*}$. Then there exists $y \in(K O)^{\sim}(X / Y)$ such that $p^{*} y=x$ and

$$
\mathrm{g} \cdot \operatorname{dim} y \leqq \max \{1+\operatorname{dim} Y, \mathrm{~g} \cdot \operatorname{dim} x\} .
$$

Proof. Let $m=\max \{1+\operatorname{dim} Y, g \cdot \operatorname{dim} x\}$. Then there exists an $m$-plane bundle $\xi$ over $X$ whose stable class is $x$. From the exact sequence

$$
(K O) \sim(Y) \stackrel{i^{*}}{\leftarrow}(K O) \sim(X) \stackrel{p^{*}}{\leftarrow}(K O) \sim(X / Y)
$$

where $i^{*}$ is induced by the inclusion $i: Y \subset X$, it follows that $i^{*} x=0$, and so $i^{*} \xi$ is stably trivial. But since $m>\operatorname{dim} Y, i^{*} \xi$ must be trivial. Hence, from the exact sequence of sets

$$
[Y, B O(m)] \stackrel{i^{*}}{\leftarrow}[X, B O(m)] \stackrel{p^{*}}{\leftarrow}[X / Y, B O(m)]
$$

Received by the editors February 18, 1966.

1 This paper is based on a portion of the author's doctoral dissertation submitted to the Department of Mathematics at the University of Chicago, June 1965. 
there exists an $m$-plane bundle $\eta$ over $X / Y$ such that $p^{*} \eta=\xi$. Take $y$ to be the stable class of $\eta$.

Lemma 2. Let $X$ be the suspension of a finite $C W$-complex (or, more generally, a co-H-space with homotopy co-unit and co-inverse). Then for each $n \geqq 0,\left\{x \in(K O)^{\sim}(X) \mid\right.$ g.dim $\left.x \leqq n\right\}$ is a subgroup of $(K O)^{\sim}(X)$.

Proof. Let $x$ and $y$ be the stable classes of $n$-plane bundles $\xi$ and $\eta$, respectively, over $X$. Then $x+y$ is the stable class of $\xi \oplus \eta$. Let $f, g: X \rightarrow B O(n)$ be classifying maps for $\xi$ and $\eta$, respectively. The following diagram is homotopy commutative:

$$
\begin{aligned}
& X \stackrel{\Delta}{\rightarrow} X \times X \stackrel{f \times g}{\longrightarrow} B O(n) \times B O(n) \rightarrow B O(2 n)
\end{aligned}
$$

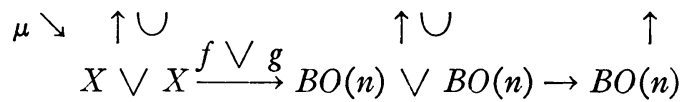

Here $\Delta$ is the diagonal map, $\mu$ is the pinch map, $B O(n) \bigvee B O(n)$ $\rightarrow B O(n)$ is the folding map, and $B O(n) \times B O(n) \rightarrow B O(2 n), B O(n)$ $\rightarrow B O(2 n)$ are induced by the inclusions $O(n) \times O(n) \subset O(2 n), O(n)$ $C O(2 n)$, respectively. The composition of the maps of the top row is a classifying map for $\xi \oplus \eta$. Since it factors through $B O(n), \xi \oplus \eta$ is equivalent to the Whitney sum of an $n$-plane bundle with a trivial bundle. Hence g.dim $(x+y) \leqq n$.

Since $[X, B O(n)]$ is a group under track multiplication, given $f: X \rightarrow B O(n)$, there exists $g: X \rightarrow B O(n)$ such that the composition

$$
X \rightarrow X \vee X \stackrel{f \vee g}{\longrightarrow} B O(n) \vee B O(n) \rightarrow B O(n)
$$

is homotopically trivial. Hence given an $n$-plane bundle $\xi$ over $X$, the above diagram implies that there exists an $n$-plane bundle $\eta$ over $X$ with $\xi \oplus \eta$ trivial. Hence g.dim $(-x) \leqq n$.

Proof of Theorem. $P^{n} / P^{m}$ is the Thom space of $(m+1) \gamma_{n-m-1}$ over $P^{n-m-1}$. Since $m+1>n-m-1$, this bundle admits a nowhere zero cross-section, and so $P^{n} / P^{m}$ is a suspension.

If $i: P^{m} C P^{n}$ is the standard inclusion, $i^{*} x_{n}=x_{m}$. By [1], $2^{\phi(m)} r x_{m}$ $=0$, and so $2^{\phi(m)} r x_{n}$ is in the image of $p^{*}$, where $p: P^{n} \rightarrow P^{n} / P^{m}$ denotes the natural projection. By Lemma 1 , there exists $y \in(K O) \sim\left(P^{n} / P^{m}\right)$ such that $p^{*} y=2^{\phi(m)} r x_{n}$, and g.dim $y \leqq \max \left\{m+1, \operatorname{g.dim}\left(2^{\phi(m)} r x_{n}\right)\right\}$. By Lemma 2, g.dim $s y \leqq$ g.dim $y$ for any integer $s$. Hence

$$
\text { g.dim } p^{*} s y \leqq \max \left\{m+1, \operatorname{g.dim}\left(2^{\phi(m)} r x_{n}\right)\right\} \text {. }
$$

Applications. (i) g.dim $(16 s+7) x_{13}=7$ for all integers $s$.

(ii) g.dim $(16 s+8) x_{15}=8$ for all integers $s$. 
Proof. It follows from the fact that $P^{15}$ admits 8 everywhere linearly independent vector fields [1] that g.dim $16 x_{15} \leqq 7$. Hence g.dim $16 x_{13} \leqq 7$. Applying the Theorem with $n=13, m=6, r=2$, we obtain g.dim $\left(16 s x_{13}\right) \leqq 7$. By [3, Lemma 2.2], g.dim $\left(q x_{r}\right) \leqq k$ if and only if $g \cdot \operatorname{dim}(-q+k) x_{r} \leqq k$. Hence, g.dim $(-16 s+7) x_{13} \leqq 7$. The reverse inequality follows by a Stiefel-Whitney class argument. Since $s$ is arbitrary, replacing $s$ by $-s$ yields (i).

(ii) is obtained similarly, applying the Theorem with $n=15$, $m=7$, and $r=1$.

\section{REFERENCES}

1. J. F. Adams, Vector fields on spheres, Ann. of Math. (2) 75 (1962), 603-632.

2. M. F. Atiyah, Immersions and embeddings of manifolds, Topology 1 (1962), 125-132.

3. B. J. Sanderson, A non-immersion theorem for real projective spaces, Topology 2 (1963), 209-211.

The University of Chicago and

The University of California, Berkeley 